\title{
The muon to electron conversion process and the Mu2e experiment at Fermilab
}

\author{
Stefano Miscetti *i \\ Laboratori Nazionali di Frascati of INFN \\ Via Enrico Fermi 40, 00044, Frascati (Roma), Italy \\ E-mail: Stefano.Miscetti@Lnf.Infn.It
}

The Mu2e experiment at Fermilab will measure the charged-lepton flavor violating (CLFV) neutrino-less conversion of a negative muon into an electron in the field of an aluminium nucleus. CLFV processes in the muon sector have negligible rates in the Standard Model. However, in many Beyond the Standard Model scenarios their rates are within the reach of the next generation of experiments. The goal of the Mu2e experiment is to improve by four orders of magnitude the best previous measurement and reach a single event sensitivity of $2.5 \times 10^{-17}$ on the conversion rate with respect to the muon capture rate. The distinctive signature of muon to electron conversion is a mono-energetic electron with momentum very close to the muon rest mass. In this paper, we explain the sensitivity to the scale of new physics scale. We then discuss the Mu2e experimental technique, based on four elements: the high muon intensity, the pulsed beam structure, the extinction of out of time particles and precise electron identification in the detector solenoid. The production and transport of the muons employs a sophisticated magnetic system composed pf a production, a transport and a detector solenoid. The experiment's goal is achieved by stopping $10^{18}$ low momentum muons on an aluminium target in three years of running. This paper focuses on the design and status of the experiment.

The European Physical Society Conference on High Energy Physics

5-12 July, 2017

Venice

\footnotetext{
*Speaker.

${ }^{\dagger}$ On behalf of the Mu2e Collaboration.
} 


\section{CLFV processes in the muon channel}

Although the Standard Model (SM) is very well tested in many regimes, it likely appears to be incomplete. In the SM, hadronic flavor transitions are allowed while in the lepton sector (with massless neutrinos) transitions between charged and neutral leptons preserve flavor. Nevertheless, neutrino oscillations have been observed thus implying massive neutrinos and non-conservation of lepton flavor number in the neutral sector. Even assuming neutrino oscillations, charged lepton flavor violation (CLFV) processes are extremely suppressed in the $\operatorname{SM}\left(\operatorname{BR}(\mu \rightarrow e \gamma) \leq 10^{-52}\right.$ [1]) while, in many of Beyond the Standard Model (BSM) scenarios (SUSY, Leptoquarks, GUT, etc.), rates for CLFV processes are within reach of the next generation of experiments ( [2]).

CLFV searches in muon channels are of particular interest because of their high rates and the possibility of carrying out clean measurements free of hadronic corrections in the calculation. No CLFV process has been observed yet, and because of the negligible background above, any experimental detection would be a clear indication of BSM physics. Experimentally, the search for CLFV in muon channels $(\mu \rightarrow e \gamma, \mu \rightarrow 3 e$, and muon to electron conversion, $\mu N \rightarrow e N$, in the presence of a nucleus) is progressing extremely well. The sensitivity reach has provided stringent limits for both $\mu \rightarrow e \gamma\left(\mathrm{BR}<4.2 \times 10^{-13}\right.$ at $90 \%$ C.L. [3]) and muon-to-electron conversion on gold $\left(R_{\mu e}<7 \times 10^{-13}[4]\right)$. A solid international program exists with the upgrade of MEG underway [5], a proposed $\mu \rightarrow 3 e$ experiment, Mu3e, [6] at PSI and with the approved programs on the muon to electron conversion at FNAL (Mu2e) [7] and J-PARC (COMET/DeeMe) [8, ?].

\section{Mu2e: search for $\mu$ to $e$ conversion at FNAL}
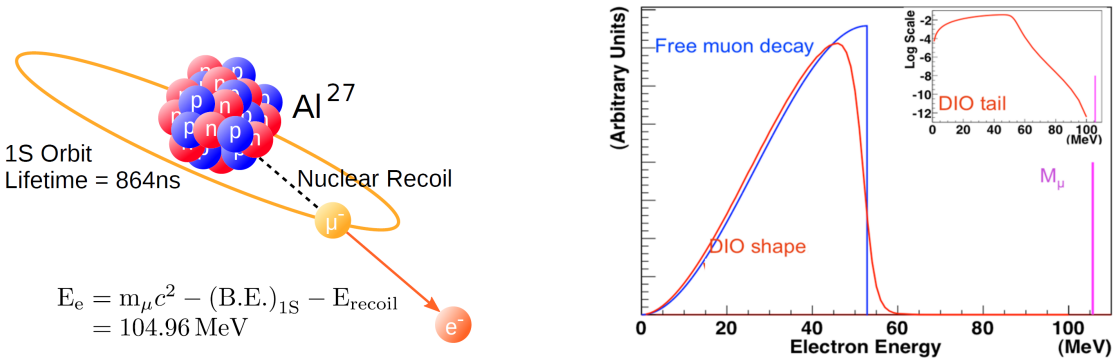

Figure 1: (Left) Sketch of the muon to electron conversion process. (Right) Energy spectrum for electrons produced from muon decays in orbit. An ideal resolution is assumed.

The measurement of the neutrinoless $\mu^{-}$to $\mathrm{e}^{-}$conversion rate, $R_{\mu e}$ is defined relative to the ordinary muon capture on nucleus. The conversion electron (CE) signature consists of a monoenergetic electron (Fig. 1(left)), with momentum close to the muon rest mass. Stopped muons on aluminium have a $39 \%$ of undergoing a 3 body decay when orbiting around the nucleus (mиоn decay in orbit, DIO). The DIO electron spectrum substantially differs from that of free decay (with the Michel edge at $52.8 \mathrm{MeV}$ ) due to the presence of a large recoil tail that falls rapidly as the electron energy approaches the kinematical endpoint. The CE line has to be distinguished, with a high momentum resolution detector, from the DIO electron spectrum shown in Fig. 1(right). The 
goal of Mu2e is to achieve a single CE event sensitivity of $2.5 \times 10^{-17}$, or constrain the $R_{\mu}$ value to below $8 \times 10^{-17}$ at $90 \%$ C.L. Apart from the DIO contribution, additional background sources were observed in the previous best experiment, Sindrum-II [4]. A prompt background coming from radiative pion capture (RPC, $\pi+N \rightarrow \gamma+N^{\prime}$ ) was found to be a limiting uncertainty. In the RPC, the electron positron pair, produced either by internal or external conversion, becomes a source of fake $\mathrm{CE}$ candidates when the $\mathrm{e}^{-}$momentum is in the right selection window. These considerations have driven the design strategy of the new muon to electron conversion experiments (Mu2e, COMET) to improve the limit from Sindrum-II by four orders of magnitude.

There are four ingredients needed to achieve this improvement: (i) a high intensity muon beam. The goal is to increase the muon intensity by $10^{4}$ to reach $10^{11}$ muons/sec on target. This is obtained combining a high rate particle production and a curved solenoidal system to create a transport channel that selects both charge and momentum. (ii) A pulsed beam structure to reject prompt background. Mu2e has selected an aluminium target where the muon lifetime in the bound system $\left(\tau_{\mu}=864 \mathrm{~ns}\right.$ ) well matches the bunch period of the Fermilab accelerator (micro-bunch of $1694 \mathrm{~ns}$ period). The trick is to wait for the prompt backgrounds to decay and start the data acquisition $\sim 700 \mathrm{~ns}$ after the bunch arrival time. (iii) A proton extinction better than $10^{-10}$. The number of protons traveling in the beam in the out of time window has to be reduced to the indicated level with respect to the in time protons. (iv) A redundant high-precision detector. This is needed to analyse the products from muon interaction on target to separate CE and DIO spectra and make the contribution from additional background sources negligible. The layout of

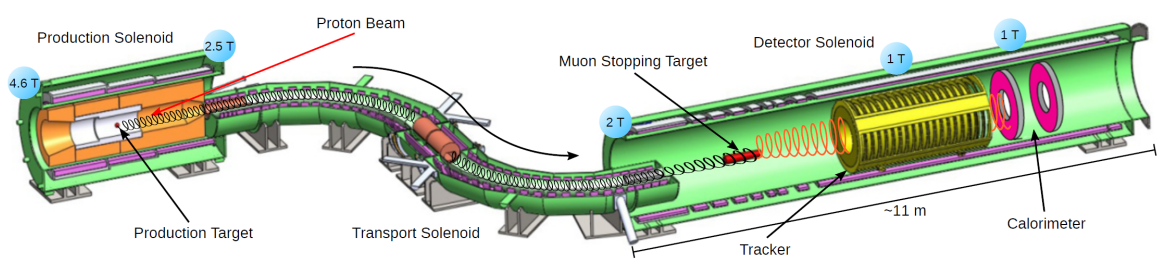

Figure 2: Layout of the Mu2e experiment.

the Mu2e experiment is shown in Fig. 2. A series of superconducting solenoids forms a graded magnetic system composed of a Production Solenoid, PS, a Transport Solenoid, TS, and a Detector Solenoid, DS. The PS contains a tungsten target that is struck by an $8 \mathrm{GeV}$ pulsed proton beam. A gradient field in the PS (from 2.5 to 4.6 Tesla as shown) acts as a magnetic lens to focus the produced low energy particles (pions, muons and a small number of antiprotons) into the transport channel. The S-shaped Transport Solenoid efficiently transfers low energy, negatively charged particles to the end of the beamline while allowing a large fraction of pions to decay into muons. Positive and negatively charged particles drift in opposite directions while traveling through the curved solenoidal field, and a mid-section collimator removes nearly all the positively charged particles (leaving a small antiproton residual background). The DS uses a graded field from 2 to 1 Tesla in the upstream region where the stopping target resides to increase acceptance for CE events. An uniform magnetic field of 1 Tesla occupies the region of the tracker and calorimeter systems. Approximately $50 \%$ of the muon beam is stopped by the target; the surviving beam stops on the 
beam dump at the end of the cryostat. A muon stopping rate of $10 \mathrm{GHz}$ allows the experiment to reach the final goal of $6 \times 10^{17}$ stopped muons on target in 3 years of running. Muons that stop in the aluminium target are captured in an atomic excited state and promptly cascade to the $1 \mathrm{~S}$ ground state with $39 \%$ decaying in orbit and the remaining $61 \%$ captured by the nucleus. Low energy photons, neutrons and protons are emitted in the nuclear capture process. These make up an irreducible source of accidental activity that is the origin of a large neutron fluence on the detection systems. Together with the flash of particles accompanying the beam, the capture process produces the bulk of the ionizing dose observed in the detector system and its electronics. The tracking
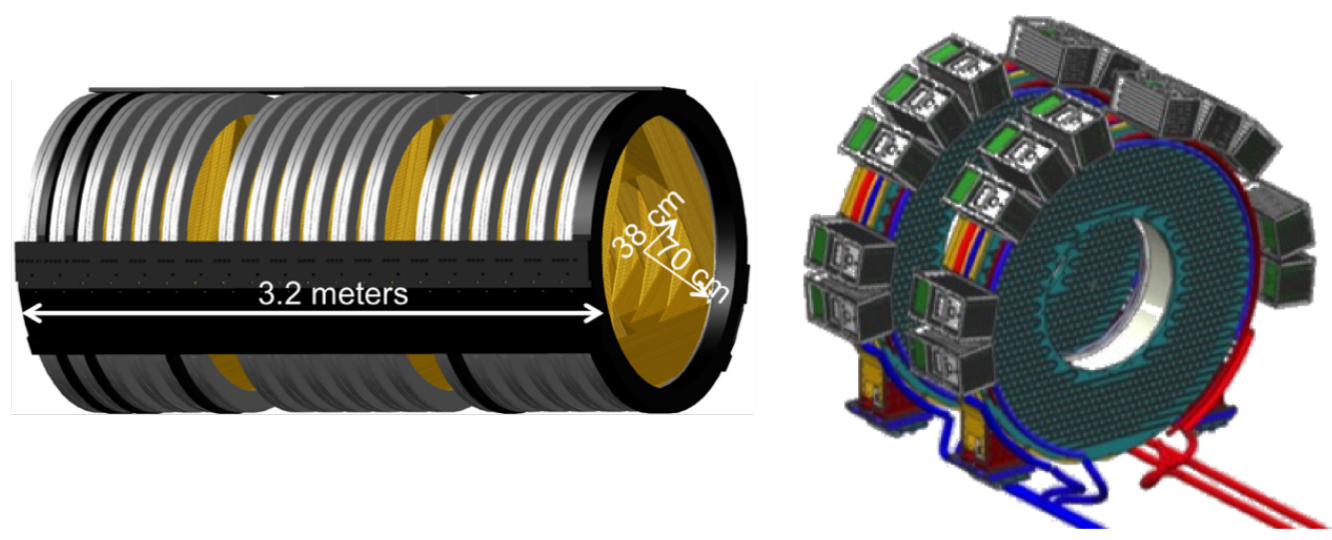

Figure 3: (Left) Sketch of the Mu2e straw tracker system. (Right) Sketch of the calorimeter system.

detector [9] is composed of nearly 20,000 low mass straw drift tubes oriented transversally to the solenoid axis and arranged in 18 stations as shown in Fig. 3(left). Each tube is $5 \mathrm{~mm}$ in diameter and contains a $25 \mu \mathrm{m}$ sense wire. The straw walls are made out of Mylar and have a thickness of $15 \mu \mathrm{m}$. The tracker measures the momenta of the charged particles by reconstructing their trajectories from the hits detected in the straws. The circular hole inside the tracker makes it insensitive to charged particle momenta at or below the Michel edge since the particle orbit radius is too small. The electromagnetic calorimeter system [10] is composed of two disks of undoped CsI scintillating crystals and is placed downstream of the tracker. Each CsI crystal is readout by two UV extended Silicon Photomultipliers. In Fig. 3(right) a drawing of the disks is shown. The calorimeter should provide powerful particle rejection between muons and electrons, an independent trigger system and a seed for tracking reconstruction. To fulfill these requirements, the calorimeter must have an energy resolution of $\mathrm{O}(5 \%)$ and a time resolution $<500 \mathrm{ps}$, for $100 \mathrm{MeV}$ electrons. A full description of the tracking and calorimeter systems can be found in another report of these proceedings.[11] The major background source in Mu2e is due to cosmic ray muons that produce fake CE candidates when interacting with the detector materials. These events occur at a rate of approximately one/day. In order to reduce their contributions in the experiment lifetime, the external area of the DS, and a part of the TS, are covered by a Cosmic Ray Veto (CRV) system.[12] The requirement for the CRV system is to obtain a veto efficiency of at least $99.99 \%$ for cosmic ray tracks while withstanding an intense radiation environment. Comprised of four staggered layers of scintillation slabs (Fig. 4(right)), the CRV counters are read out with two embedded wavelength 
shifting fibers, each one in optical contact with a $2 \times 2 \mathrm{~mm}^{2}$ Hamamatsu SIPM. Test beams on full size prototype have been carried out demonstrating that the needed light yield can be reached. Irradiation of SiPMs with neutrons have also been done to understand the maximum level of fluence acceptable for operations.

At $100 \mathrm{MeV}$, the momentum resolution is dominated by fluctuations in the energy loss in the stopping target and bremsstrahlung in the tracker, with the trajectory altered by multiple scattering. The resolution for CE tracks is well-parametrised by a Crystal Ball function with a negative bremsstrahlung tail, a gaussian core of $116 \mathrm{keV}$ and a long exponential positive resolution tail. The finite resolution has a large effect on the DIO falling spectrum that translates to a residual contamination in the signal region. Fig. 4 (right) shows the signal and background distributions as seen by a full simulation of the experiment (pileup included) in the following conditions: (i) $3.6 \times 10^{20}$ protons on target (ii) a corresponding number of $6 \times 10^{17}$ muon stops and (iii) a $R_{\mu e}$ of $2 \times 10^{-16}$. A momentum window is selected by maximising the signal over background. A DIO contribution of 0.14 events is found. An estimate of the other background contributions is presented in Tab. 2, for a total background of 0.46 events. The number of reconstructed candidate events is 6.66. This counting corresponds to set a limit on $R_{\mu e}$ below $8 \times 10^{-17}$ at $90 \%$ C.L., in good agreement with the experiment's design goal. The Mu2e experiment has, as of this writing, successfully procured
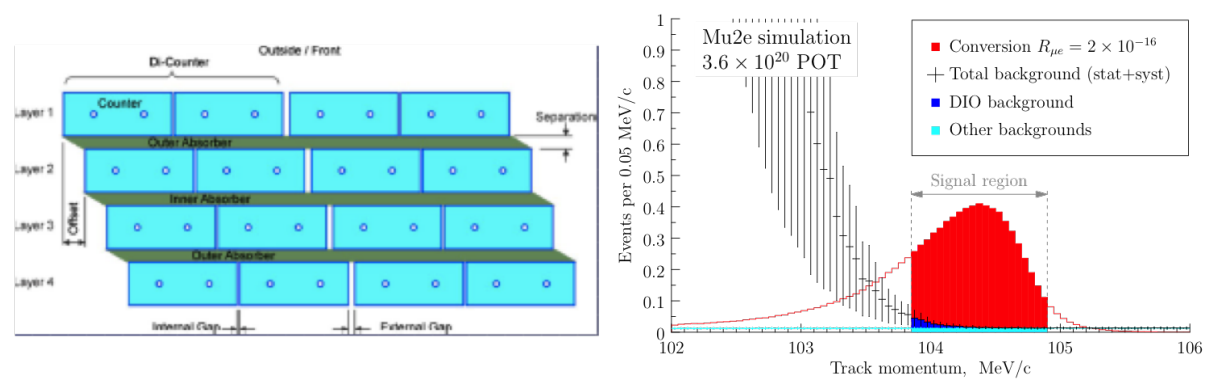

Figure 4: (Left) Structure of the CRV counters. (Right) Distribution of electron momentum spectrum for simulated DIO and CE events.

all superconducting cables, completed civil construction and obtained CD-3 (Critical Decision 3) from DOE. CD-3 grants permission to start the construction for the accelerator, the magnetic system, the muon beam line and all the detector components. The heart of the Mu2e apparatus is provided by the superconducting magnetic system whose design, fabrication, assembly and commissioning drives the experiment schedule. The status of the magnet as well as the construction and testing of the superconducting cables is satisfactory. An international bid for the DS and PS has been concluded and the construction phase for the large magnets is started at General Atomics, San Diego, USA. For the TS, after the construction of one module prototype by ASG Superconducting, in collaboration with the INFN group of Genova, the contract has been awarded to ASG. Construction of the 52 coils is progressing well, with the first 32 already being completed. The first TS module, assembled with two coils, will be delivered in the fall and then fully qualified at the HAB test facility in Fermilab. The construction of the detectors is beginning at the moment of writing. The schedule foresees a completion of the installation of detectors and commissioning 


\begin{tabular}{lll}
\hline Category & Background process & Estimated Yield (events) \\
\hline Intrinsic & Decay in orbit (DIO) & $0.14 \pm 0.11$ \\
& Muon Capture (RMC) & $0.000 \pm 0.004$ \\
Late Arriving & Pion capture (RPC) & $0.025 \pm 0.003$ \\
& Muon decay in flight & $<0.003$ \\
& Pion decay in flight & $0.000_{-0.000}^{+0.001}$ \\
& beam electrons & $(2.5 \pm 1.2) \times 10^{-4}$ \\
Miscellaneous & pbar & $0.047 \pm 0.024$ \\
& Cosmic rays & $0.24 \pm 0.071$ \\
\hline & Total & $0.46 \pm 0.13$ \\
\hline
\end{tabular}

Table 1: Expected background list as evaluated by full simulation at CD3.

with cosmic rays at the end of 2020 .

\section{Acknowledgments}

We are grateful for the vital contributions of the Fermilab staff and the technical staff of the participating institutions. This work was supported by the US Department of Energy; the Italian Istituto Nazionale di Fisica Nucleare; the US National Science Foundation; the Ministry of Education and Science of the Russian Federation; the Thousand Talents Plan of China; the Helmholtz Association of Germany; and the EU Horizon 2020 Research and Innovation Program under the Marie Sklodowska-Curie Grant Agreement N.690385. Fermilab is operated by Fermi Research Alliance, LLC under Contract No. De-AC02-07CH11359 with the US Department of Energy, Office of Science, Office of High Energy Physics.

\section{References}

[1] S.M.Bilenky et al., Phys. Lett. B67, p 309 (1977)

[2] F.Grancagnolo et al., Nucl.Phys.Proc.Supp. 248-250, p 1-146 (2014).

[3] A.M.Baldini et al. (MEG collaboration), Eur. Phys. Journal C - Vol 76 (8), p 434 (2016).

[4] The SINDRUM II Collaboration, Eur.Phys.J. C47, p 337-346 (2006)

[5] A. Baldini et al. (MEG collaboration), arXiv:1301.7225 (2013).

[6] https://www.psi.ch/mu3e/DocumentsEN/ResearchProposal.pdf (2012).

[7] The Mu2e Collaboration, FERMILAB-TM-2594, arXiv:1501.05241 (2015).

[8] http://comet.kek.jp/Documents_files/PAC-TDR-2016/COMET-TDR-2016_v2.pdf (2016).

[9] https://doi.org/10.1016/j.nuclphysbps.2014.02.010.

[10] MyeongJae Lee (Mu2e collaboration), Nuclear and Particle Physics Proceedings, Vol 273-275, p 2530-2532 (2016).

[11] N.Atanov et al., Nucl. Instr. and Meth. A 824 p 695-698, 10.1016/j.nima.2015.09.074 (2016).

[12] S. Giovannella (Mu2e collaboration), The Mu2e tracking and calorimeter systems, these proceedings.

[13] A. Artikov et al. (Mu2e CRV group), arXiv:1511.00374 (2015). 